\title{
Pathogenic Mechanisms of HIV Disease: The Role of Viral Replication and Immune Activation Anthony S Fauci*ł
}

\author{
Address: National Institute of Allergy and Infectious Diseases, National Institutes of Health, Building 31, Room 7A-03, Bethesda, MD 20892-2520, \\ USA \\ Email: Anthony S Fauci* - afauci@niaid.nih.gov \\ * Corresponding author $\ddagger$ Presenting author
}

from 2005 International Meeting of The Institute of Human Virology

Baltimore, USA, 29 August - 2 September 2005

Published: 8 December 2005

Retrovirology 2005, 2(Suppl I):SI09 doi:I0.II86/I742-4690-2-SI-SI09

HIV replication and the immune response to the virus lead to a state of generalized immune activation, which drives the pathogenesis of HIV disease. HIV-induced immune activation results in increased T-cell turnover (production and destruction), increased death of T cells, a decline in the size of the CD4+ T-cell pool, and a state of activation-induced immunodeficiency. In many HIVinfected individuals, these phenomena can be reversed or mitigated with effective antiretroviral therapy (ART), which blunts HIV replication and can reduce plasma levels of viremia to undetectable levels. Recent data from our laboratory have demonstrated fundamental differences between viremic and aviremic individuals with regard to the physiologic state of the resting CD4+ T-cell reservoir of HIV and the phenotype and function of T cells, B cells and natural killer (NK) cells. In terms of the CD4+ T-cell reservoir, resting cells in viremic versus aviremic individuals differentially express certain genes associated with HIV replication and are continually poised to express virus as a result of continual activation related to viremia; thus, true latency likely does not exist in these patients. We had previously reported that much greater stability exists in aviremic individuals, suggesting the presence of a truly latent reservoir of virus in this population. However, recent data indicate that the normal physiologic process of low-level immune activation in aviremic individuals sustained the low level turnover and propagation of the latent HIV reservoir. Previously, we have demonstrated that HIV-mediated hyperactivation induces the terminal differentiation of $\mathrm{B}$ cells. More recently, we have determined that certain B-cell genes are upregulated in HIVviremic individuals as compared with HIV-aviremic individuals. Many of these genes are associated with terminal B-cell differentiation and death by apoptosis. Recent data indicate that there is a balance between expression of survival and apoptotic genes in HIV-infected individuals that is impacted by the presence and level of viremia. In viremic individuals we also have characterized a population of aberrantly activated CD56- NK cells that manifest lower-than-normal expression of Natural Cytotoxicity Receptors and a normal or increased expression of inhibitory NK receptors. This dichotomy is reflected in decreased cytotoxic function and decreased secretion of TNF-alpha and interferon-gamma. In addition, NK cells from viremic individuals express Fas on their surfaces at significantly increased levels, and are more susceptible to apoptosis upon exposure to sFASL. Furthermore, viremia impacts the NK cell-dendritic cell interactions that are critical to normal immune responsiveness. Taken together, our data indicate that HIV pathogenesis involves both direct and indirect effects of HIV and a relentless cycle of aberrant immune activation that drives the disease process in HIV-infected individuals. 